\title{
Bio-Inspired Adhesive Systems for Next-Generation Green Manufacturing
}

\author{
Hoon Yi', Insol Hwang', Minho Sung', Dael Lee', Jeong-Hun Kim², Seong Min Kang², \\ Won-Gyu Bae ${ }^{3}$, and Hoon Eui Jeong ${ }^{1, \#}$ \\ 1 Department of Mechanical Engineering, Ulsan National Institute of Science and Technology (UNIST), Eonyang-eup, Ulju-gun, Ulsan, South Korea, 689-798 \\ 2 School of Mechanical and Aerospace Engineering, Seoul National University, Daehak-dong, Gwanak-gu, Seoul, South Korea, 151-742 \\ 3 Interdisciplinary Program of Bioengineering, Seoul National University, Daehak-dong, Gwanak-gu, Seoul, South Korea, 151-742 \\ \# Corresponding Author / E-mail: hoonejeong@unist.ac.kr, TEL: +82-52-217-2339, FAX: +82-52-217-2409
}

KEYWORDS: Biomimetics, Gecko, Dry adhesive, Microstructure, Precision manufacturing

\begin{abstract}
Inspired by the remarkable adhesion properties of gecko lizards, bio-inspired dry adhesives with smart adhesion properties have been developed in the last decade. Compared to earlier dry adhesives, the recently developed ones exhibit excellent adhesion strength, smart directional adhesion, and structural robustness. With these unique adhesion properties, bio-inspired dry adhesive pads have strong potential for use in precision industries such as semiconductor or display manufacturing. In this communication, we present a new manufacturing technology based on advanced dry adhesive systems that enable precise manipulation of large-area substrates over repeating cycles without any requirement for external force application. This new manufacturing technique is also highly accurate and environment-friendly, and thus has strong potential as a next-generation clean manufacturing technology.
\end{abstract}

Manuscript received: June 19, 2014 / Accepted: July 8, 2014

\section{NOMENCLATURE}

SOI wafer $=$ silicon-on-insulator wafer

PDMS $=$ polydimethylsiloxane

DRIE $=$ deep reactive ion etching

$\mathrm{PECVD}=$ plasma enhanced chemical vapor deposition

$\mathrm{Si}=$ silicon

$\mathrm{SR}=$ spacing ratio

\section{Introduction}

The attachment pads of gecko lizards represent one of the most versatile and effective adhesive structures in nature, reflected in their exceptional ability to walk freely on various surfaces including vertical walls and even ceilings without any surface contamination. ${ }^{1,2}$ This unusual adhesion capability is attributed to arrays of millions of microscopic foot hairs that split into hundreds of smaller nanoscale ends called spatulas..$^{3-8}$ Inspired by the fascinating adhesion properties of gecko lizards, artificial dry adhesives with superior and smart adhesion properties have been developed over the last decade with the aid of micro- and nanoscale manufacturing techniques. ${ }^{5-7,9-12}$ Compared to earlier dry adhesives, the recently developed ones show remarkable adhesion strength, repeatable and reversible adhesion, rough surface adaptation, and directional adhesion. . $^{5,9,10,13}$

Therefore, now it is time to drive fundamental research on bioinspired dry adhesives into a new epoch by developing unique and practical applications for these materials. Among their many possible applications in various aspects of the daily life as well as in industries, dry adhesives have strong potential for use in precision industries such as semiconductor or display manufacturing, where clean and residuefree transportation of fragile silicon or glass substrates is required during the product-assembly process. ${ }^{5,6}$ During the assembly process of semiconductors or display devices, silicon wafers or glass substrates, which are usually very thin, large, and fragile, should be carefully handled with high accuracy. Moreover, there should be no surface contamination on the substrates. ${ }^{5,6}$ Thus, dry adhesive technology has strong potential to replace existing techniques such as vacuum suction or electrostatic chucks since dry adhesives enable precise manipulation of various substrates without any surface contamination in a reversible, repeatable, and durable manner. Furthermore, dry adhesive technology does not require external forces (e.g., vacuum or electrostatic forces) 
for the attachment of substrates, which will enable cost-effective and environment-friendly manufacturing in precision industries.

Here, we introduce a new manufacturing technology based on advanced dry adhesive systems that enable precise manipulation of various substrates over tens of thousands of repeating cycles without any requirement for external force application. This new manufacturing technique is also highly accurate and environment-friendly, and thus has strong potential as a next-generation clean manufacturing technology.

\section{Fabrication of Gecko-Inspired Dry Adhesives}

To develop a new manufacturing system that enables precise manipulation and transportation of large-area substrates, we utilized bio-inspired, mushroom-like micropillar arrays. These arrays present an efficient form for a dry adhesive, since both the normal and shear adhesion strength can be significantly enhanced owing to the presence of a thin spatulate structure, ${ }^{14,15}$ which maximizes the adhesive area in contact with the target surface. ${ }^{10,16-19}$ Furthermore, they are relatively easy to produce in a scalable manner and also mechanically more durable as compared with hierarchical nanostructures. ${ }^{6}$

Fig. 2 shows a schematic procedure for fabricating bio-inspired dry adhesives with mushroom-like micropillars by a series of surface micromachining steps and replica molding of PDMS. First, a SOI wafer with microholes was prepared by photolithography and subsequent DRIE. The SOI wafer (p-type, 100) possessed a 1- $\mu \mathrm{m}$-thick oxide layer on a $500-\mu \mathrm{m}$-thick silicon layer. A $20-\mu \mathrm{m}$-thick top silicon layer was then formed over the oxide layer. Additionally, an 800-nmthick $\mathrm{SiO}_{2}$ layer was deposited on top of the silicon layer for realizing enhanced etching resistivity during DRIE by PECVD. Here, the $1-\mu \mathrm{m}-$ thick oxide layer imbedded between the top and bottom Si substrates acted as an etch-stop layer. DRIE in the vertical direction was carried out to pattern microhole arrays until the complete exposure of the etchstop layer was achieved. Subsequent over-etching in the lateral direction resulted in negative patterns of mushroom-shaped micropillars on the SOI wafer (Fig. 3(a)). For replica molding, the patterned wafer was treated with trichloro $(1 \mathrm{H}, 1 \mathrm{H}, 2 \mathrm{H}, 2 \mathrm{H}-$ perfluorooctyl) silane. After the SOI master was prepared, PDMS replicas were prepared by molding the fabricated SOI master with a PDMS prepolymer. A 10:1 mixture of the PDMS prepolymer (Sylgard 184A) and curing agent (Sylgard 184B) was poured onto the SOI master. Then, it was put into a convection oven and cured for $2 \mathrm{~h}$ at $70^{\circ} \mathrm{C}$. After curing, the PDMS replicas were carefully removed from the master to avoid possible fracture of the spatulate tips.

Fig. 3(b) shows a photograph of the replicated dry adhesives in which four different kinds of mushroom-shaped microstructures are embedded: micropillars with (i) $17 \mu \mathrm{m}$ tip diameter, $15 \mu \mathrm{m}$ post diameter, and $30 \mu \mathrm{m}$ space (spacing between neighboring pillars); (ii) $22 \mu \mathrm{m}$ tip diameter, $20 \mu \mathrm{m}$ post diameter, and $40 \mu \mathrm{m}$ space; (iii) $17 \mu \mathrm{m}$ tip diameter, $15 \mu \mathrm{m}$ post diameter, and $15 \mu \mathrm{m}$ space; and (iv) $22 \mu \mathrm{m}$ tip diameter, $20 \mu \mathrm{m}$ post diameter, and $20 \mu \mathrm{m}$ space. The height of the micropillars was about $20 \mu \mathrm{m}$, and each micropillar had a patterned area of $3 \times 3 \mathrm{~cm}^{2}$. Fig. 3(c) shows the representative SEM image of the resulting microstructures. As shown, a mushroom-shaped micropillar

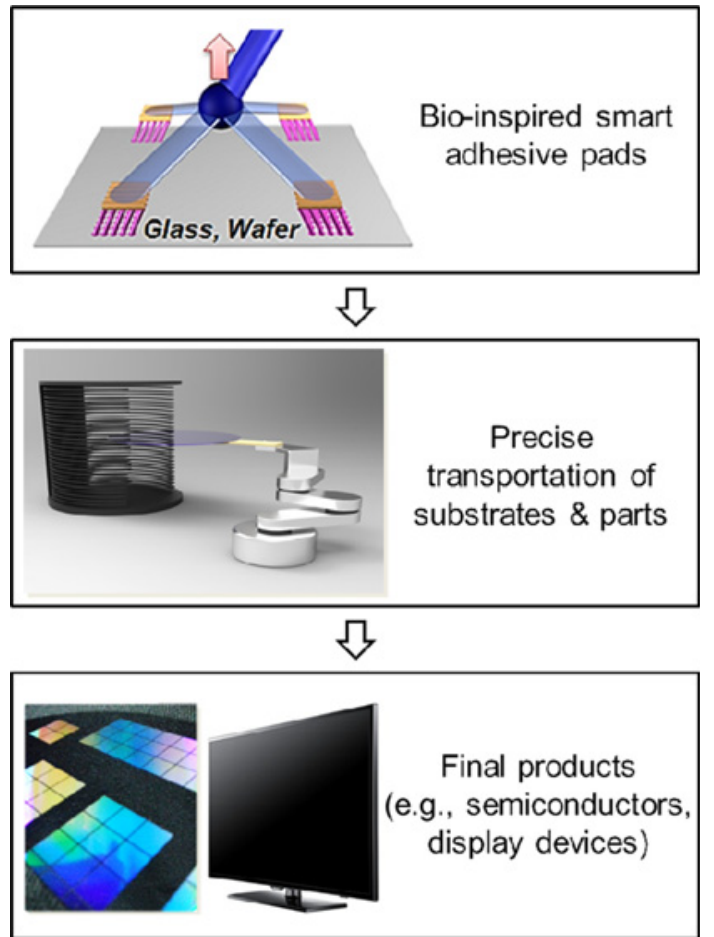

Fig. 1 Examples of applications of bio-inspired dry adhesives in precision manufacturing

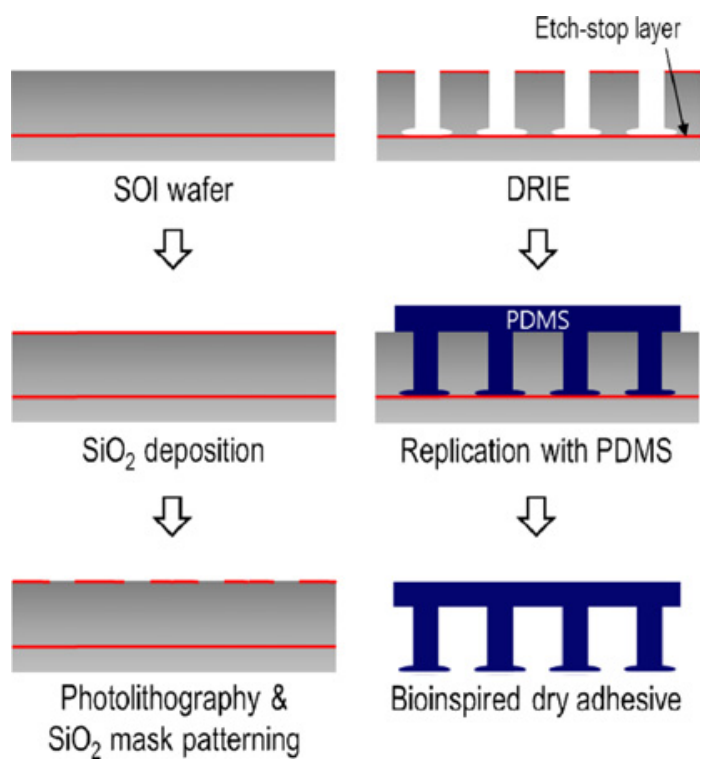

Fig. 2 Schematic illustration of fabricating bio-inspired dry adhesives with mushroom-like microstructures

array (22 $\mu \mathrm{m}$ tip diameter, $20 \mu \mathrm{m}$ post diameter, and $20 \mu \mathrm{m}$ space) was successfully fabricated with good structural integrity and uniformity. Specifically, the spatulate tip of the microstructures, which plays a significant role in enhancing adhesion strength, was successfully fabricated.

\section{Results and Discussions}

\subsection{Evaluation of Adhesion Strength}

The pull-off forces of PDMS micropillars were evaluated by 
(a)

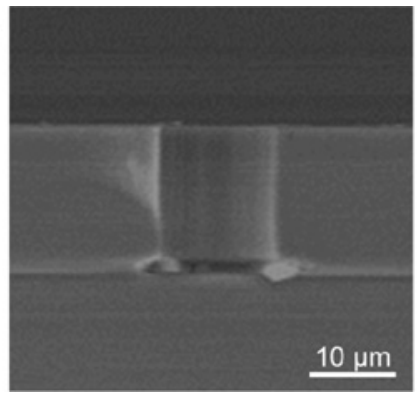

(b)

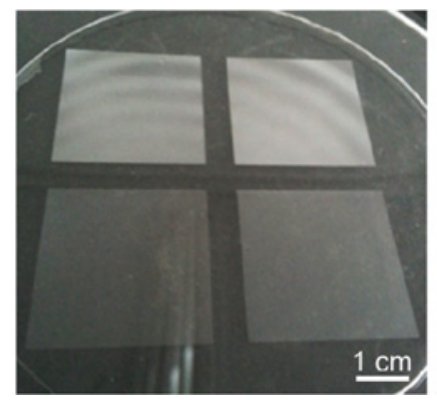

(c)

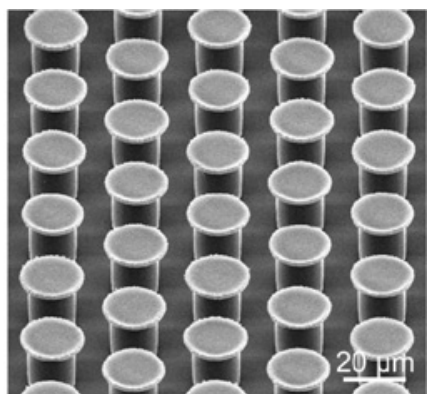

Fig. 3 (a) A cross-sectional SEM image of etched negative patterns of mushroom-shaped micropillars on the SOI wafer. (b) A photograph showing the fabricated dry adhesive film having four different mushroom-like micropillars. (c) An SEM image of fabricated mushroom-shaped micropillar array (22 $\mu \mathrm{m}$ tip diameter, $20 \mu \mathrm{m}$ post diameter, and $20 \mu \mathrm{m}$ space)

(a)

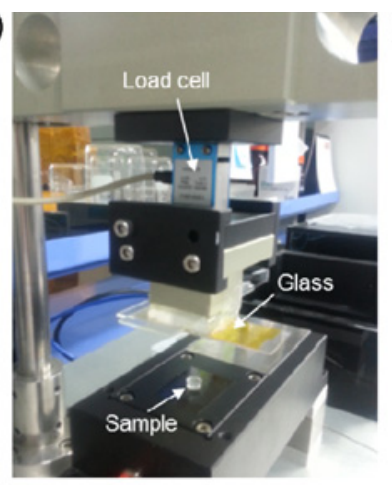

(b)

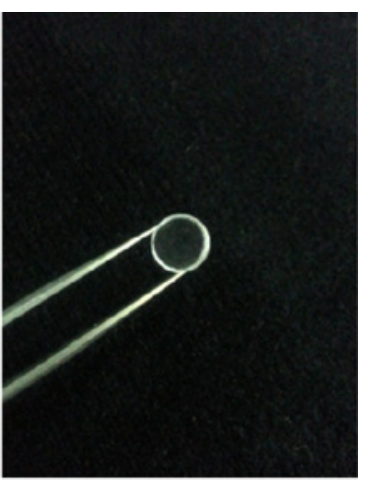

(c)

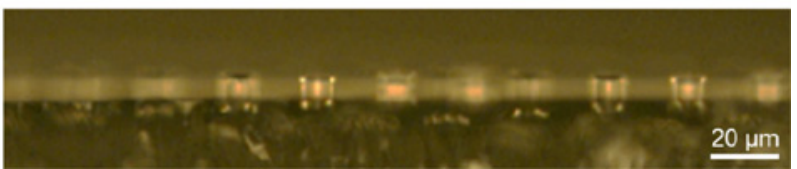

Fig. 4 (a) A custom-built adhesion measurement system. The system is composed of a motorized driving part with a load cell moving in vertical direction and a flat stage. (b) A photograph showing the circular dry adhesive pad (diameter $=7 \mathrm{~mm}$ ). (c) An optical microsopy image showing the cross-section of dry adhesive pad with mushroom-like microstructures

custom-built equipment at a relative humidity of $40 \%$ and an ambient temperature of $25^{\circ} \mathrm{C}$ (Fig. 4(a)). The equipment consisted of motorized load cells and a flat stage. The load cell (333AL, KTOYO, Korea) was used for measuring the adhesion force in the $\mathrm{z}$ (vertical) direction. A circular dry adhesive patch (Fig. $4(\mathrm{~b})$, diameter $=7 \mathrm{~mm}$, thickness $=2 \mathrm{~mm}$ ) was attached to a flat glass surface bonded to the load cell under a controlled preload ranging from 2 to $70 \mathrm{~N}(2 \mathrm{~N}, 5 \mathrm{~N}, 10 \mathrm{~N}, 20 \mathrm{~N}, 30 \mathrm{~N}$, $40 \mathrm{~N}, 50 \mathrm{~N}, 60 \mathrm{~N}$, and $70 \mathrm{~N}$ ), and an in-plane strain was applied by the motorized stage until separation occurred. The adhesion measurement was carried out 30 times for each sample under identical conditions.

As shown in Fig. 5, the pull-off forces of PDMS micropillars were maximized under a preload of $2 \mathrm{~N}$ and then decreased with increasing preload. The maximum adhesion force was as high as $\sim 4.0 \mathrm{~N}$ $\left(\sim 10.4 \mathrm{~N} \mathrm{~cm}^{-2}\right)$ for a micropillar array with $15 \mu \mathrm{m}$ post diameter and $15 \mu \mathrm{m}$ space. The adhesion strength was reduced to $\sim 0.6 \mathrm{~N}\left(\sim 1.7 \mathrm{~N} \mathrm{~cm}^{-2}\right)$ when the preload increased to $70 \mathrm{~N}$. The decrease in the pull-off strength with increasing preload may be caused by the displacement of the micropillars due to the lateral deformation of the back film by the Poisson effect under an excessive preload. ${ }^{20}$ Interestingly, the adhesion strength of dry adhesives can be accurately controlled by changing the

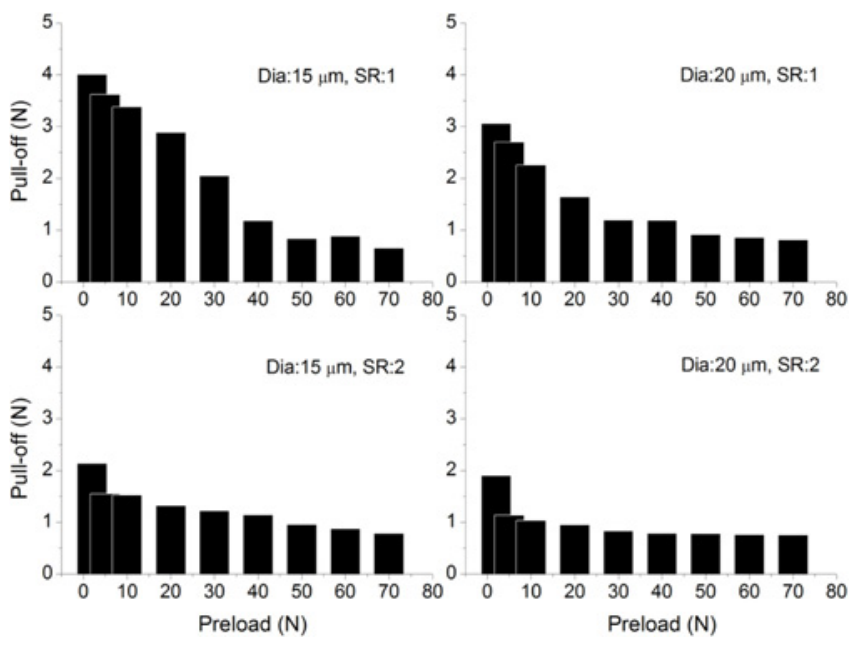

Fig. 5 Measurement of pull-off strength of fabricated mushroom-like micropillars as a function of applied preload

pillar geometries and preload, as shown in Fig. 5, which is highly useful for the development of precise substrate transportation systems. For example, we can tune and optimize the pull-off strength of substrate transportation systems depending on the types of target substrates and process requirements.

\subsection{Application to Automated Transportation System with Dry Adhesive Pads}

The fabricated bio-inspired adhesive pads have great potential to replace existing substrate transportation techniques including vacuum suction, mechanical clamping, electrostatic chucks, etc as dry adhesion mechanisms have several distinct advantages over other ones, which enable precise manipulation of various substrates with no surface contamination in a reversible and repeatable manner (see Table 1). ${ }^{5,6,20}$ Furthermore, dry adhesive technology is cost-effective and environment-friendly as it does not require external forces (e.g., vacuum or electrostatic forces) for the attachment of substrates.

To demonstrate the practical applications of dry adhesives in precision manufacturing, we developed a wafer transport system by integrating the adhesive pads with an automated robotic system. Fig. 6 shows the automated transportation system based on dry adhesives. Three circular dry adhesive pads (diameter $=7 \mathrm{~mm}$, thickness $=2 \mathrm{~mm}$ ) were attached to a robotic arm that could move in the $\mathrm{z}, \mathrm{R}$, and $\theta$ directions. The robotic arm equipped with adhesive pads approached a 
Table 1 Advantages and disadvantages of various transportation techniques

\begin{tabular}{ccc}
\hline Techniques & Holding force & $\begin{array}{c}\text { Prevention of } \\
\text { contamination \& dama }\end{array}$ \\
\hline Dry adhesive & $\bullet \bullet \bullet$ & $\bullet \bullet \bullet$ \\
\hline $\begin{array}{c}\text { Vacuum chuck } \\
\text { Mechanical clamping }\end{array}$ & $\bullet \bullet \bullet$ & $\cdots$ \\
\hline Electrostatic chuck & $\cdots$ & $\cdots$ \\
\hline (a) & \\
\hline
\end{tabular}

Fig. 6 (a) A schematic illustration of the automated wafer transportation system utilizing (b) a robotic arm equipped with bioinspired dry adhesive pads

silicon wafer (diameter: $300 \mathrm{~mm}$ ) and formed a conformal contact between the microstructures on the pad and the substrate. After forming a firm attachment between the dry adhesive and substrate, the automated system started to move and transport the silicon wafer without any sliding or detachment. After arriving at a target location (stackable wafer cassette), the arm started to move downward, releasing the wafer from the adhesive with the aid of a cassette guide.

Upon repeating the attachment and detachment test, the wafer transportation system based on dry adhesive pads exhibited superior transportation capabilities such as no surface contamination or damage, firm attachment, easy detachment, and a repeatability of $>10,000$ cycles (data not shown).

\section{Conclusions}

In summary, we have presented a bio-inspired dry adhesive in the form of mushroom-shaped micropillar arrays and demonstrated an automated substrate transportation system based on the adhesive pads. The automated robotic system equipped with adhesive pads enabled precise and stable manipulation of a $300 \mathrm{~mm}$ Si wafer over 10,000 repeating cycles without any requirement for external force application. It is envisioned that the new approach presented here could prove to be a next-generation clean and environment-friendly transportation technique for precision manufacturing.

$\bullet \bullet \bullet \bullet \bullet \bullet \bullet \bullet \bullet \bullet ⿺ 辶 ⿻$

Excellent Good $\quad$ Fare $\quad$ Poor Prevention of
substrate bending $\quad$ Scalability $\quad$ Energy saving $\quad$ Cost saving

$\begin{array}{lccc}\bullet \bullet \bullet & \bullet \bullet \bullet & \bullet \bullet \bullet & \bullet \bullet \bullet \\ \bullet & \bullet \bullet & \bullet \bullet \bullet & \bullet \bullet \\ \bullet \bullet & \bullet \bullet & \bullet & \bullet\end{array}$
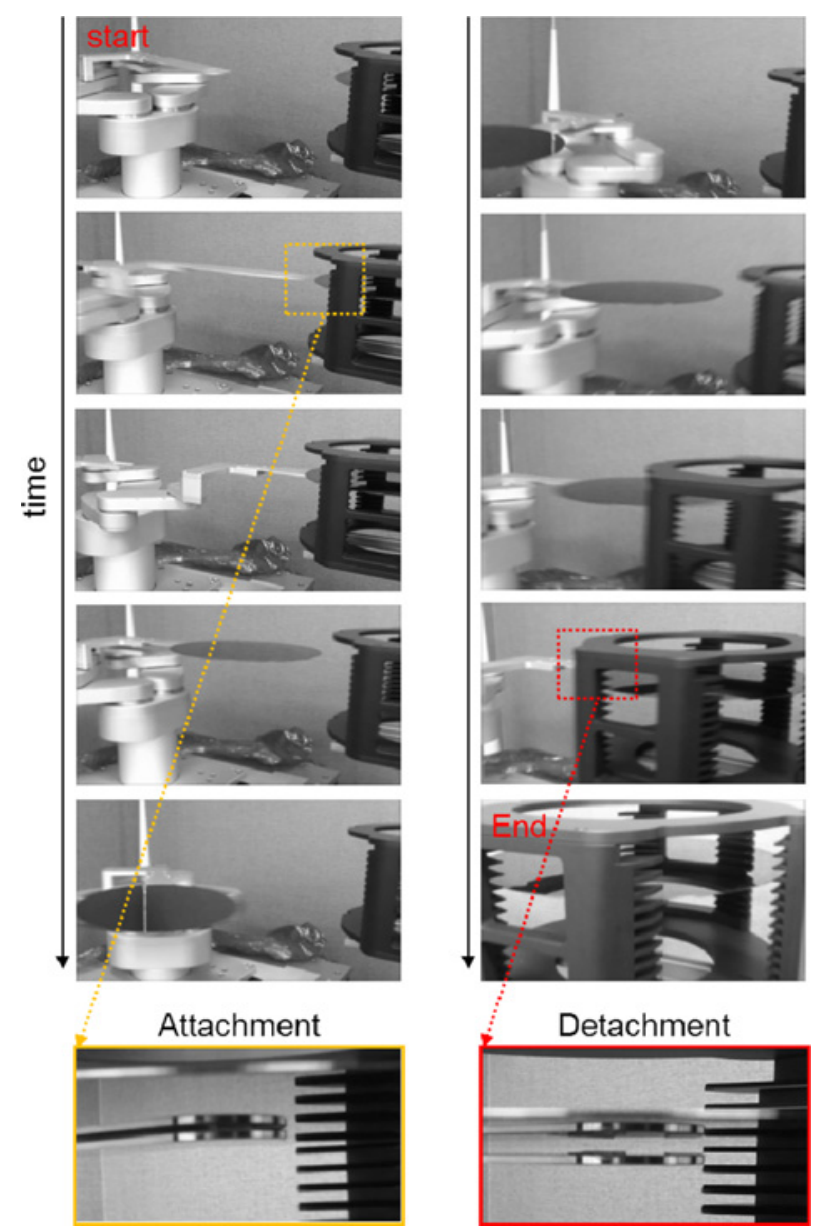

Fig. 7 Time-lapse images showing the demonstration of automated $300 \mathrm{~mm}$ wafer transportation based on smart dry adhesives

\section{ACKNOWLEDGEMENT}

This work was supported by the Ulsan National Institute of Science and Technology (UNIST) through the 2012 Research Fund (1.120067.01) and the Creativity and Innovation Project Program (Grant UMI 1.140012.01). This work was also supported by the MOTIE/KEIT Program (Grant 10045070) and the Young Researchers Supporting Program through the National Research Foundation of Korea (NRF) (2013R1A1A1061219).

\section{REFERENCES}

1. Autumn, K., Liang, Y. A., Hsieh, S. T., Zesch, W., Chan, W. P., et 
al., "Adhesive Force of a Single Gecko Foot-Hair," Nature, Vol. 405, No. 6787, pp. 681-685, 2000.

2. Autumn, K., Sitti, M., Liang, Y. A., Peattie, A. M., Hansen, W. R., et al., "Evidence for van der Waals Adhesion in Gecko Setae," Proc. of the National Academy of Sciences, Vol. 99, No. 19, pp. 1225212256, 2002.

3. Arzt, E., Gorb, S., and Spolenak, R., "From Micro to Nano Contacts in Biological Attachment Devices," Proc. of the National Academy of Sciences, Vol. 100, No. 19, pp. 10603-10606, 2003.

4. Federle, W., "Why are So Many Adhesive Pads Hairy?" Journal of Experimental Biology, Vol. 209, No. 14, pp. 2611-2621, 2006.

5. Jeong, H. E. and Suh, K. Y., "Nanohairs and Nanotubes: Efficient Structural Elements for Gecko-Inspired Artificial Dry Adhesives," Nano Today, Vol. 4, No. 4, pp. 335-346, 2009.

6. Jeong, H. E., Lee, J.-K., Kim, H. N., Moon, S. H., and Suh, K. Y., "A Nontransferring Dry Adhesive with Hierarchical Polymer Nanohairs," Proc. of the National Academy of Sciences, Vol. 106, No. 14, pp. 5639-5644, 2009.

7. Pang, C., Kwak, M. K., Lee, C., Jeong, H. E., Bae, W.-G., and Suh, K. Y., "Nano Meets Beetles from Wing to Tiptoe: Versatile Tools for Smart and Reversible Adhesions," Nano Today, Vol. 7, No. 6, pp. 496-513, 2012.

8. Kwak, J.-S. and Kim, T.-W., "A Review of Adhesion and Friction Models for Gecko Feet,” Int. J. Precis. Eng. Manuf., Vol. 11, No. 1, pp. 171-186, 2010.

9. Jeong, H. E., Lee, S. H., Kim, P., and Suh, K. Y., "Stretched Polymer Nanohairs by Nanodrawing," Nano Letters, Vol. 6, No. 7, pp. 1508-1513, 2006.

10. Del Campo, A., Greiner, C., Álvarez, I., and Arzt, E., "Patterned Surfaces with Pillars with Controlled 3D Tip Geometry Mimicking Bioattachment Devices," Advanced Materials, Vol. 19, No. 15, pp. 1973-1977, 2007.

11. Cho, K.-J., Koh, J.-S., Kim, S., Chu, W.-S., Hong, Y., and Ahn, S.H., "Review of Manufacturing Processes for Soft Biomimetic Robots,” Int. J. Precis. Eng. Manuf., Vol. 10, No. 3, pp. 171-181, 2009.

12. Chu, W.-S., Kim, C.-S., Lee, H.-T., Choi, J.-O., Park, J.-I., et al., "Hybrid Manufacturing in Micro/Nano Scale: A Review," Int. J. Precis. Eng. Manuf.-Green Tech., Vol. 1, No. 1, pp. 75-92, 2014.

13. Chu, B., Jung, K., Han, C.-S., and Hong, D., “A Survey of Climbing Robots: Locomotion and Adhesion,” Int. J. Precis. Eng. Manuf., Vol. 11, No. 4, pp. 633-647, 2010.

14. Jeong, H. E. and Suh, K.-Y., "Precise Tip Shape Transformation of Nanopillars for Enhanced Dry Adhesion Strength," Soft Matter, Vol. 8, No. 19, pp. 5375-5380, 2012.

15. Carbone, G., Pierro, E., and Gorb, S. N., "Origin of the Superior Adhesive Performance of Mushroom-Shaped Microstructured Surfaces," Soft Matter, Vol. 7, No. 12, pp. 5545-5552, 2011.
16. Kim, S. and Sitti, M., "Biologically Inspired Polymer Microfibers with Spatulate Tips as Repeatable Fibrillar Adhesives," Applied Physics Letters, Vol. 89, No. 26, p. 261911, 2006.

17. Jeong, H. E., Kwak, M. K., and Suh, K. Y., "Stretchable, AdhesionTunable Dry Adhesive by Surface Wrinkling," Langmuir, Vol. 26, No. 4, pp. 2223-2226, 2010.

18. Kwak, M. K., Jeong, H. E., Bae, W. G., Jung, H. S., and Suh, K., "Anisotropic Adhesion Properties of Triangular-Tip-Shaped Micropillars,” Small, Vol. 7, No. 16, pp. 2296-2300, 2011.

19. Heepe, L., Kovalev, A. E., Filippov, A. E., and Gorb, S. N., "Adhesion Failure at 180000 Frames Per Second: Direct Observation of the Detachment Process of a Mushroom-Shaped Adhesive," Physical Review Letters, Vol. 111, No. 10, Paper No. 104301, 2013.

20. Bae, W.-G., Kim, D., and Suh, K.-Y., "Instantly Switchable Adhesion of Bridged Fibrillar Adhesive via Gecko-Inspired Detachment Mechanism and Its Application to a Transportation System,” Nanoscale, Vol. 5, No. 23, pp. 11876-11884, 2013. 